\title{
Simulation study on the vehicle mobility model based on the SUMO Xue Yuan ${ }^{\mathrm{a}}$, Bai Yun ${ }^{\mathrm{b}}$, Chunming $\mathrm{Li}^{\mathrm{c}}$ \\ Institude of information Engineering,Inner Mongolia University of Technology,Huhhot,Inner Mongolia, 01008 ,China

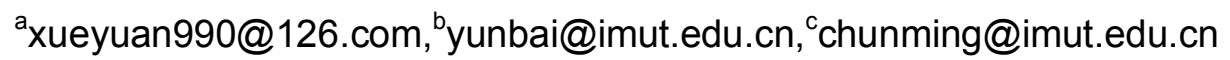

\begin{abstract}
Key words: Vehicular Ad Hoc Networks, Mobility models, SUMO
Abstract. Simulation is one of the common technologies to study the Vehicular Ad Hoc Networks (VANET), and different simulation models obtain the different simulation results. Aimed at this situation, this paper compares and analyzes the Krauss model and RDM model, and constructs the simulation system based on the SUMO. Moreover, the AODV protocol is used to verify the influence of the two kinds of models on the routing protocol on this basis. The experimental results show that the performance of Krauss model is better than that of the RDM model in the AODV routing protocol, and the different mobile models have different effects on the simulation results of the routing protocol.
\end{abstract}

\section{Introduction}

VANET is a kind of special mobile self-organized network which is applied in the vehicles. The vehicles within the scope of a certain communication can mutually exchange their information such as speed, position, etc. and the data perceived by the vehicle sensor, and can connect each other automatically to build a mobile network[1].

VANET simulation model is different from other general simulation models. It is a mobility model based on its basic idea that there is safe distance limit among the cars and special requirements, which more conforms to the actual road requirements[2]. This paper structures the simulation environment which is even much closer to the practical one by using urban traffic simulation platform SUMO[3], auxiliary tools MOVE, and network simulation platform NS2, and then AODV protocol is used to verify the influence of different mobile models on the routing protocol performance of VANET.

\section{Mobility model introduction}

Random direction mobility model (RDM).RDM is the simplest model[4]. The node of the model finds the point $S$ as the initial point in the mobile area, randomly select a direction, walks at the preset speed, stops for a period of time after reaching the border, and then continues to select the next goal to move, stop, move again, and so forth until the simulation time ends. The model can be regarded as the cycle of the following steps:

(1) Stay for a certain amount of time;

(2) Randomly select a destination in mobile area;

(3) Move to the destination at the speed within maximum speed;

(4) Repeat the above steps.

Krauss model. Krauss model is spatial continuous car following model, the current vehicle keeps a certain distance with the vehicle ahead in the model, and the safe speed allows it to adapt the speed cut behavior of the vehicle ahead[5]. It is supposed that $a$ is the maximum acceleration of vehicle $\left(\mathrm{m} / \mathrm{s}^{2}\right), b$ is the maximum deceleration of vehicle $\left(\mathrm{m} / \mathrm{s}^{2}\right), V_{\max }$ is the maximum speed of vehicle $(\mathrm{m} / \mathrm{s}), l$ is the vehicle length $(\mathrm{m}), €$ is the proficiency of the driver $(€ \in(0,1)$, the larger the value is, the more unskilled the proficiency is). The safe speed in this model is shown as follows. 


$$
v_{\text {safe }}=v_{t}(t)+\frac{g(t)-v_{t}(t) * \Gamma}{\left(v_{t}+v_{f}\right) /(2 * b)+\Gamma}
$$

Where $v_{t}(t)$ is the speed of vehicle ahead at the time $t, v_{f}(t)$ is the speed of the vehicle behind at the time $t, g(t)$ is the space between the vehicle ahead and the vehicle in front, and $\Gamma$ is the response time of the driver (usually 1 second). The calculation of the safe speed of vehicle doesn't consider the actual speed limit of the road and the actual situation of acceleration in Eq.1, so the more ideal speed should take the minimum value in the three.

$$
v_{\text {des }}=\min \left[v_{\max }, v+a * t_{l}, v_{\text {safe }}\right]
$$

It is assumed that the driver doesn't have enough proficiency to adjust the vehicle to the ideal speed, simultaneously, guarantees the vehicle speed is greater than zero in the process of driving. So the final Krauss model formula is:

$$
v(t)=\max \left[0, v_{d e s}-€ * a * \operatorname{rand}()\right]
$$

Mobility models contrast. RDM model is not suitable for the VANET as the frequently-used mobility models in the Mobility Ad Hoc Networks. Random movement of the nodes doesn't accord with the movement characteristic of vehicle nodes in the VANET. In addition, in the VANET, the vehicle nodes movement is affected by the factors such as road, traffic rules, other vehicles, and so on, which makes the nodes movement have the particularity. Relative to RDM model, Krauss model belongs to the vehicle-following model, and increases the parameters such as the safety speed and safety distance, which makes the nodes movement trajectory more close to the real vehicle movement characteristics.

\section{Simulation experiment and result analysis}

This paper uses the geo-information data derived from OpenStreetMap, edits OSM data by JOSM software, and generates simulation scene with the help of SUMO and MOVE. Then it combines the network simulation platform NS2 to conduct simulation experiment. The simulation process is shown as Figure 1.

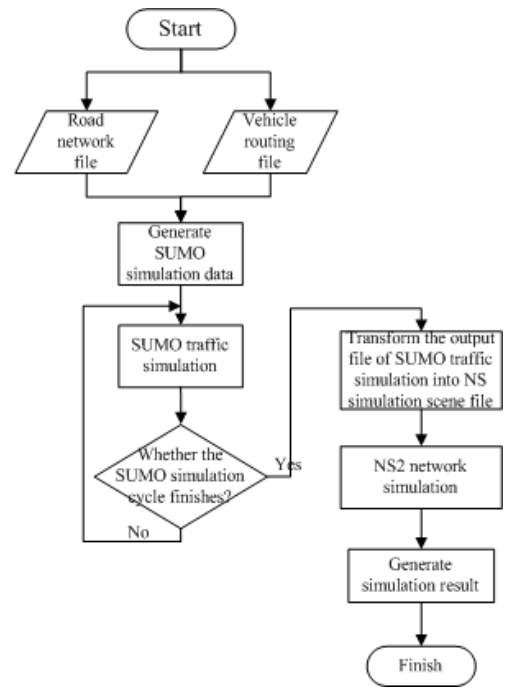

Fig.1 Flow chart of VANET simulation system

Simulation environment. This paper assesses RDM model and Krauss model by using the effect of number variation of the vehicle nodes on the Ad Hoc On-Demand Distance Vector Routing 
(AODV) routing protocol performance[6]. To reduce the error, every group of experiments will be repeated for 10 times, and then the average value will be calculated based on the experiment results.

Table 1 Experiment Parameter

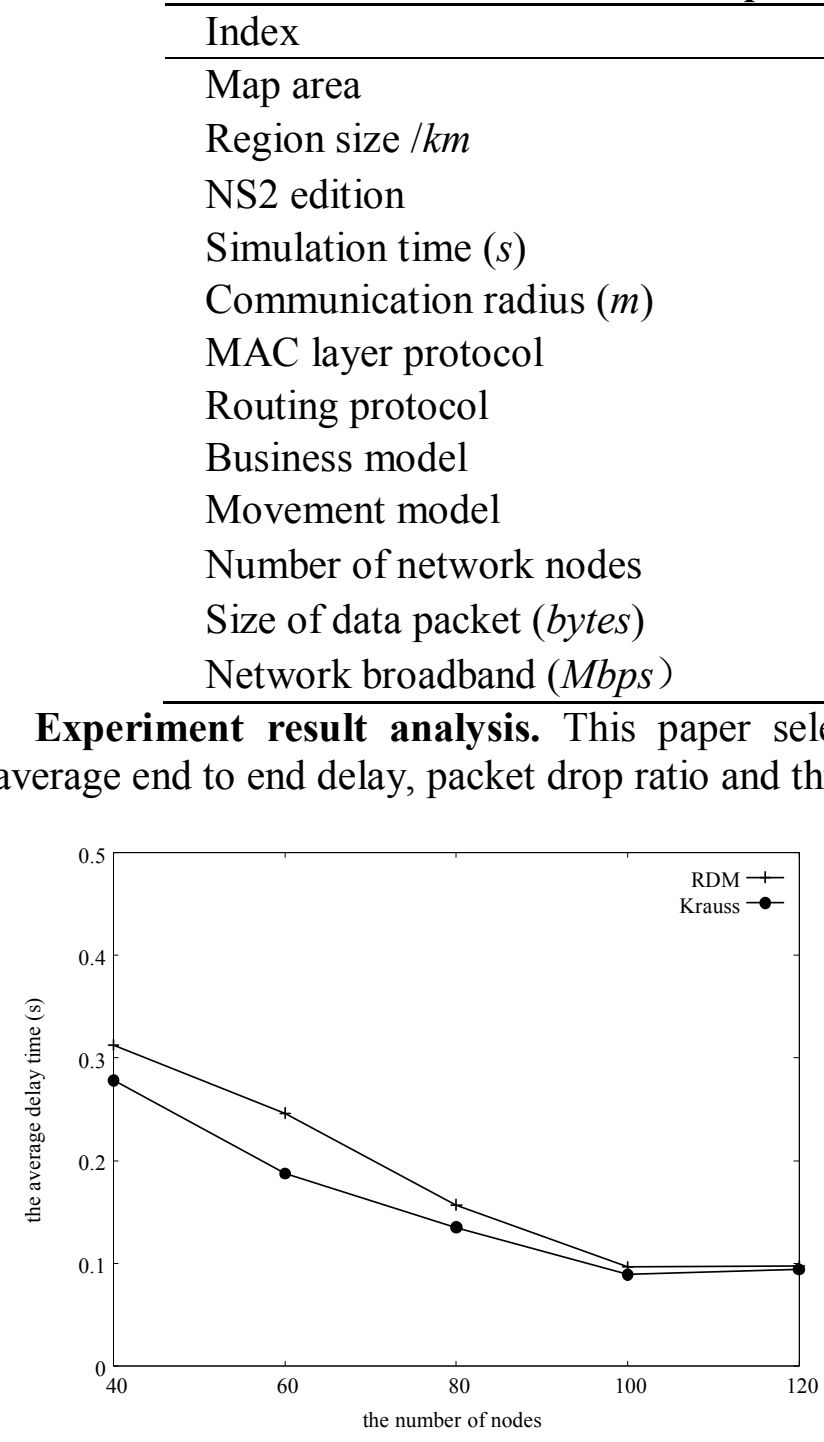

Fig. 2 The average end to end delay
Parameter

Hohhot city

$1.4 * 2.0$

2.28

1000

250

IEEE 802.11DCF

AODV

CBR

RDM、Krauss

40、60、80、100、120

1000

2

Experiment result analysis. This paper selects three routing performance indexes such as average end to end delay, packet drop ratio and throughout for analysis.

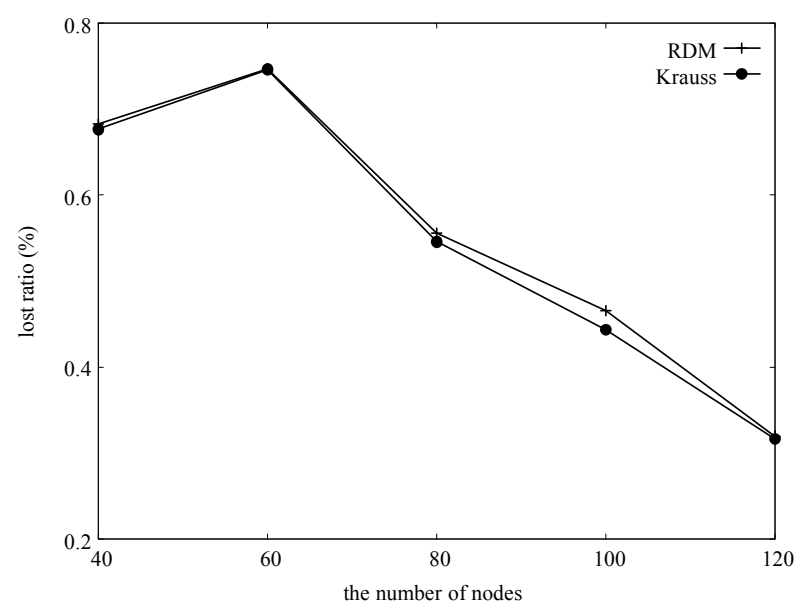

Fig. 3 The packet loss ratio

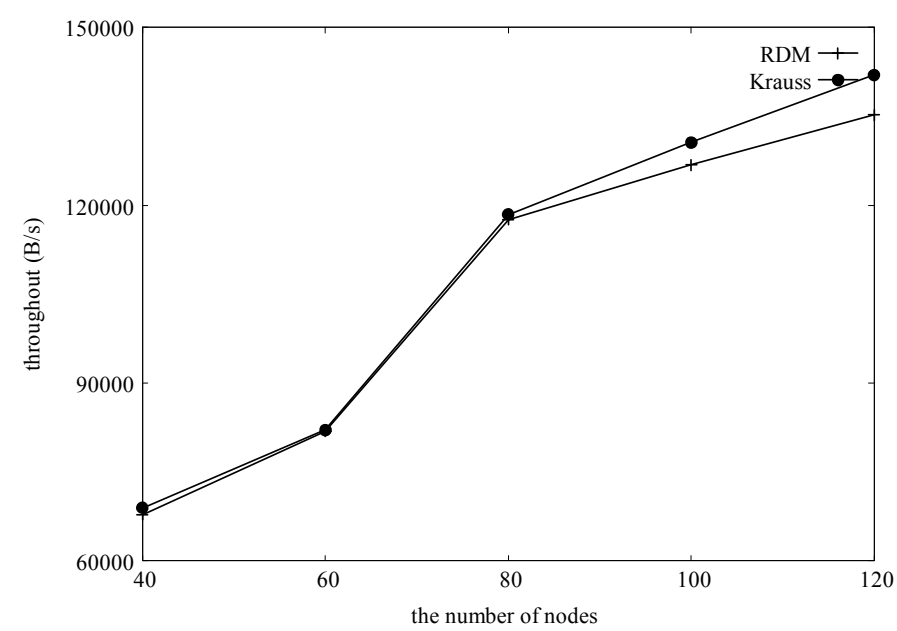

Fig. 4 The change of throughout with the node number

The average end to end delay. As shown in Fig.2, the effect of different numbers of nodes on the average end to end delay of the routing protocol. When the number of vehicle nodes in the scene ranges from 40 to 60 , the low density of the node in the road and the restriction of the node 
communication radius lead to the interruption of the network link. Even if the local repair mechanism starts, there are not enough relay nodes to repair. When the number of the vehicle nodes exceeds 100, the data delay of these two models will prolong slightly. This is because the data packet to be sent gradually becomes more with the increasing of the node number, and the transmission rate of each node data is limited. Thus the data packet will prolong the queue time in the queue of sending node, thus leading to the increasing of network delay. In VANET, the node moves fast, the routing interrupts and seeks again during the data sending, hence increasing the delay of data transmission as well.

The packet drop ratio. As shown in Fig.3, the effect of two models on the packet loss probability of AODV routing protocol. The lower the value is, the stronger the data packet delivery ability of the routing protocol is. When the number of node ranges from 40 to 60 , the node density is low and the single-hop routing is dominated, which results in the instability of the link, and quite a number of communication links would break. Therefore, the data packet can't be received by destination nodes normally. After 60 vehicle nodes, the connectivity of the vehicle communication enhances, and it is possible for long-distance vehicle to establish routing link to make communication, thus leading to the constant increasing of the proportion of the multi-hop routing in the whole routing link and continuously reduction of packet loss probability.

Throughout. As shown in Fig.4, the effect of the different node numbers on the throughout of the AODV routing protocol. The throughout reflects the number of bit the effective data grouping in the network within unit time, and expresses the ability of transmitting the effective data grouping of the whole network. Therefore, the throughout is directly related to both the average end to end delay and packet loss. The less the average end to end delay is, the lower the packet loss probability is, and the more the throughout in the same business flow is.

\section{Conclusions}

From the curve comparison among Figures 2, 3 and 4, it can be seen that Krauss model performance is superior to that of RDM model in AODV routing protocol, which is mainly caused by the two reasons, shown as follows: 1 . The topology structures are different. In RDM model, the node movement is completely random, and the mobility trajectory will not be restricted, while in Krauss model, the node movement is restricted by the topology structure of the road, moreover, the nodes distribution is relatively concentrated. 2. The node movement states are different. In RDM model, the movement state of the nodes is irrelevant to the state of the previous time, while in Krauss model, the current movement state of the node is affected by the state of the previous time. The link among adjacent nodes is relatively stable.

The experimental results show that the selection of the vehicle mobility model has an important influence on the VANET routing protocol.

\section{References}

[1] Chang cuyu, Xiang yong, Shi meilin. Present situation and the development of Vehicular Ad Hoc Networks [J]. Journal on Communications, 2007, 28(11): 116-126.

[2] Xiao ling, Li renfa, Luo juan. Vehicular Ad Hoc Networks simulation research summary [J]. System simulation, 2009, 21(17): 5330-5335, 5356.

[3] Macedo,J.; Kokkinogenis,Z.; Soares,G.; Perrotta,D.; Rossetti,R.J.F. A HLA-based multiresolution approach to simulating electric vehicles in simulink and SUMO [C].//Proc of IEEE 16th International Conference on Intelligent Transportation Systems (ITSC). The Hague:IEEE Xplore, 2013: 2367-2372

[4] E. Royer, P. M. Melliar-Smith, L. Moser.An analysis of the optimum node density for ad hoc mobile networks[C]. In Proceedings of the IEEE International Conference on Communications (ICC), 2001, 3:857-861. 
[5] Krauss S. Towards a Unified View of Microscopic Traffic Flow Theories [C] // Proc of the 8th IFAC/IFIP/IFORS Transportation Systems.UK: Pergamon, Oxford, 1997: 901- 906.

[6] PERKINS C, BELDING-ROYER E, DAS S. Ad hoc On-Demand Distance Vector (AODV) Routing[S]. IETF RFC 3561, 2003. 